\title{
EDGE PHYSICS, DIVERTORS, PUMP LIMITERS
}

\author{
K.H. Finken \\ Institut für Plasmaphysik, Forschungszentrum Jülich, Euratom Association \\ Trilateral Euregio Cluster, 52425 Jülich,Germany \\ TEL. +49 246161 5646; Fax +49 2461615452
}

\begin{abstract}
The properties of the scrape-off layer (SOL) determine both the particle removal as well as the power exhaust. The particle flows in the SOL and their radial decay give constraints for the construction of the main plasma facing components, pump limiters and divertors. Both systems allow for an adequate particle and power removal option.
\end{abstract}

\section{INTRODUCTION}

From the previous contributions we know that the core of a burning fusion reactor must provide a very good isolation against heat losses; therefore the magnetic field forms onion shell like magnetic surfaces. However, also the plasma edge on fusion reactors has to fulfil specific tasks: The power and the particles - in particular the helium ash and other impurities - coming from the core of the reactor have to be removed at an adequate rate. We know already [1] that helium particles, newly born in the fusion process, have to be pumped in a time corresponding to 5 - 10 times the energy confinement time $\tau_{\mathrm{E}}$. This high removal rate can only be obtained if the particle flux is highly compressed when it enters a pumping channel. Two separate systems capable of sufficient particle removal have been invented: the divertor and the pump limiter which are shown schematically in Figs. 1 and 2. A divertor requires special magnetic fields which modify the outer magnetic field of the reactor and allow for a particle flow into a separate chamber, the divertor chamber. The pump limiter on the other side is a simpler system than the magnetic divertor; behind limiter blades scoops are arranged in order to provide a sufficient particle compression. Unfortunately, the simple pump limiter is also less efficient in particle removal and therefore many modern tokamaks are equipped with divertors.

The desired high particle flux density into a divertor or towards a pump limiter is accompanied by an undesired strong power flux. This power flux density is at the upper tolerance limit of even the best suited materials. The incoming power density for a planned Next Step Device is estimated as follows: A reactor will have a thermal fusion power of about 2.5 - $3 \mathrm{GW}$, resulting in about $1 \mathrm{GW}$ electric power.

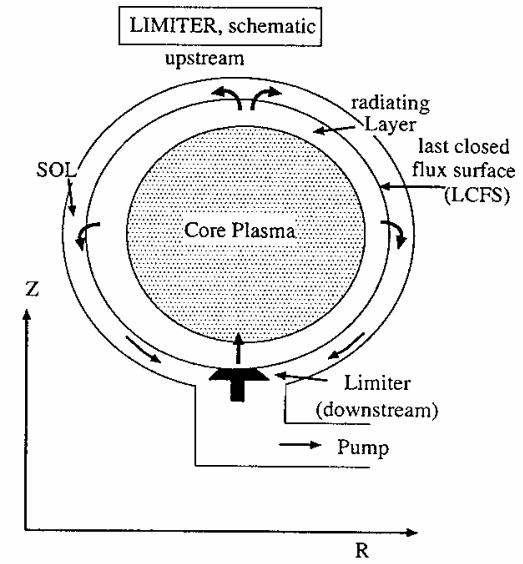

Fig. 1: Axi-symmetric (toroidal) pump limiter configuration. The typical plasma flows in the plasma boundary are also sketched.

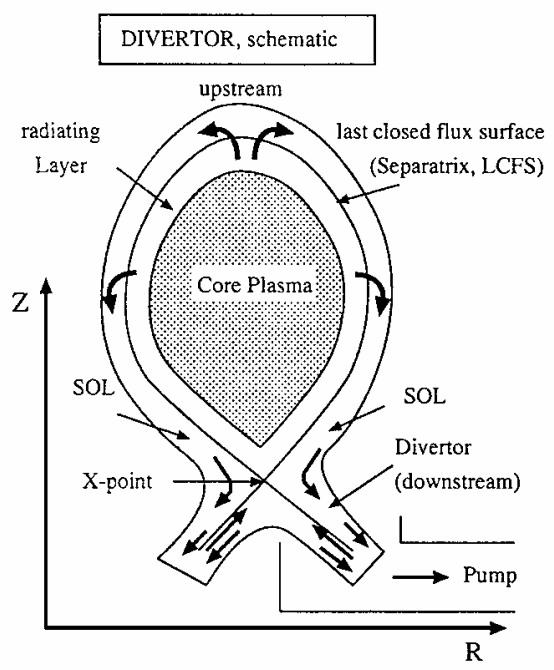

Fig. 2: Axi-symmetric (poloidal) divertor configuration with typical flow patterns (including a reversed flow near the separatrix.

$80 \%$ of this power is transferred to $14 \mathrm{MeV}$ neutrons which penetrate the first wall and deposit their power in the blanket. The remaining $20 \%$ of the power flows to the 
first wall; if the power would be deposited uniformly at the $600 \mathrm{~m}^{2}$ large area of the first wall, the average power load would amount to about $1 \mathrm{MW} / \mathrm{m}^{2}$.

The power is deposited by conduction, convection or radiation and - in contrast to the neutron power which is deposited deep into the blanket outside of the vacuum vessel this power is deposited within a few Angstroms of the wall. It is estimated that the local peak should be limited to values below $5 \mathrm{MW} / \mathrm{m}^{2}$. In addition, the energy of the incoming ions should not exceed $20 \mathrm{eV}$ in order to avoid sputtering erosion at the wall.

The requirements on particle exhaust and power deposition - for both processes the same particles come into play tend to be conflicting: For the particle collection, it is desired to guide particles to a few well defined locations at the wall, to compress them there and remove them by strong pumps; concerning material aspects, the strong heat concentration easily exceeds the level which can be cooled away without materials melting or evaporation. Unfortunately, both the sufficient pumping and the sufficient power exhaust are on a critical path of the fusion reactor and requirements on the efficiencies of both quantities cannot be relaxed. There are only very few possibilities to mitigate the power exhaust problem: one is the application of seed impurities in the plasma.

This contribution is a rather simple introduction into the basic physics of edge plasmas. Reviews on plasma edge physics are given in $[2,3,4,5,6]$ or in [7]

\section{EDGE PHYSICS}

\section{II.1 DECAY LENGTH}

The plasma in a tokamak is roughly structured in three areas: The plasma core, the gradient zone and the plasma edge. The magnetic field lines of the core and of the gradient zone are closed and are topics of the proceeding contributions: The plasma core has a relatively flat density distribution and the fusion processes will take place there; the gradient zone is determined by ionisation processes and therefore the density varies strongly in this layer. It is the transition area between edge and core; in the earlier smaller devices, the gradient zone was the largest zone in the tokamak while now with increasing tokamak size its area relatively to the core area is less dominant. The proper edge of the plasma is the so called Scrape-off Layer (SOL); in contrast to the previous two areas, the magnetic field lines here intersect material objects, namely either the limiters or the divertor target plates. Therefore these field lines are often called "open" magnetic field lines. The magnetic field line between the closed magnetic surfaces and the SOL is called the "separatrix"; in particular for divertor configurations, the separatrix forms one singular point, the X-point, where two magnetic field lines intersect.

For the plasma edge, the ratio of the transport perpendicular to the magnetic field to the one parallel to the field is a decisive quantity. In the plasma core and in the gradient zone, the perpendicular transport determines the loss rate of energy and particles resulting in energy and particle confinement times $\tau_{\mathrm{E}}$ and $\tau_{\mathrm{p}}$. The parallel transport doesn't play any role in many cases because it does not lead to a radial displacement of the particles and therefore to no enhanced loss. In contrast to these regions, the ratio of the transport coefficients determines the width of the channels in the SOL carrying particles and power to the plasma facing components and is therefore important both for the particle removal efficiency and for the heat load distribution.

The balance between parallel and perpendicular fluxes determines the width of the SOL. In the simple SOL model, the density and temperature decay laws are simple exponential functions due to the character of the diffusion differential equation. Experimentally, electron densities and temperatures can be measured by Langmuir probes or by atomic beams. As shown in Fig. 3, the data can be fit rather well by an exponential function [8]. As indicated in this figure, one also observes sometimes "bumps" in the SOL. The explanation of these effects goes beyond the assumptions made for the simple model and require e.g. ionisation effects or radial electric fields.

The ion temperature in the SOL is less known than $\mathrm{T}_{\mathrm{e}}$; collisional transfer rates between electrons and ions indicate that the ion temperature decay length is considerably larger than the one of $T_{e}$. Therefore, also the value of $T_{i}$ in the SOL should be much larger then $T_{e}$; this expectation has been verified experimentally [9]

The actual width of the SOL -typically the e-folding length - is considerably longer than expected when assuming that classical collisions determine the diffusion coefficient ( $D_{\text {class }}=r_{L}{ }^{2} / \tau_{\text {coll }} ; r_{L}=$ Larmor radius, $\tau_{\text {coll }}=$ collision time $)$. Therefore, the so-called Bohm diffusion coefficient $\left(D_{B o h m}\right.$ $=(1 / 16) * k T[\mathrm{eV}] / B[T])$ is often applied; the Bohm diffusion describes an anomalously enhanced radial loss due to electrostatic turbulence in the plasma edge. In contrast to the core plasma, the radial transport enhancement is a positive feature for the scrape-off layer because otherwise the scrape-off layer would even be narrower and the heat removal problem would increase even more. 

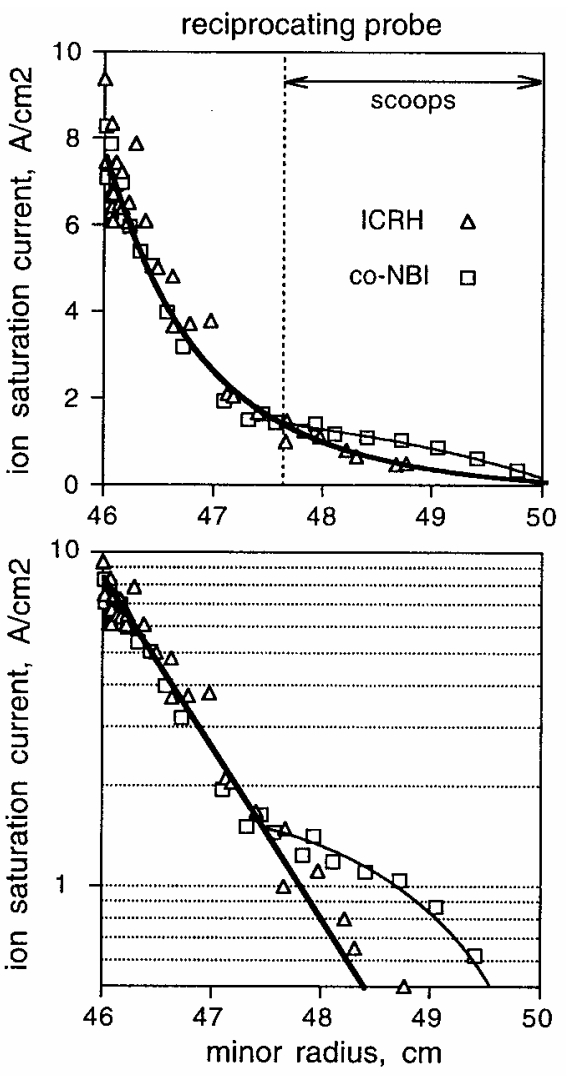

Fig. 3: Ion saturation current profiles of the SOL. The data were taken by a scanning probe. NBI and ICRF heated plasmas are compared (from [8]). The top figure is in linear scale and the bottom one in logarithmic scale.

\section{II.2 SOL - FLOW}

The parallel flow in the flux tube can easily be derived by the single fluid MHD equations. Writing continuity and momentum equations as

$\frac{\partial}{\partial x}(n \mathrm{v})=S(x) ; \quad \frac{\partial}{\partial x}\left(m n \mathrm{v}^{2}+2 n T\right)=0$

and after integration along $\mathrm{x}$, a dimensionless form is obtained:

$\hat{n} \widehat{\mathrm{v}}=F-1 ; \quad \hat{n}\left(\widehat{\mathrm{v}}^{2}+1\right)=2$

where $\hat{n}$ is normalised to the "left" tube end, $\widehat{\mathrm{v}}$ to the sound velocity $\mathrm{c}_{\mathrm{s}}$ which is assumed to be uniform along the flux tube (isothermal approximation) and

$F=\frac{1}{n_{-L} c_{s}} \int_{-L}^{x} S\left(x^{\prime}\right) d x^{\prime}$ the integration constants are chosen such that the Bohm criterium at the end of the tube is fulfilled. Continuity and momentum equations can be easily be solved:

$$
\widehat{\mathrm{v}}^{2}+\frac{2}{1-F} \widehat{\mathrm{v}}+1=0
$$
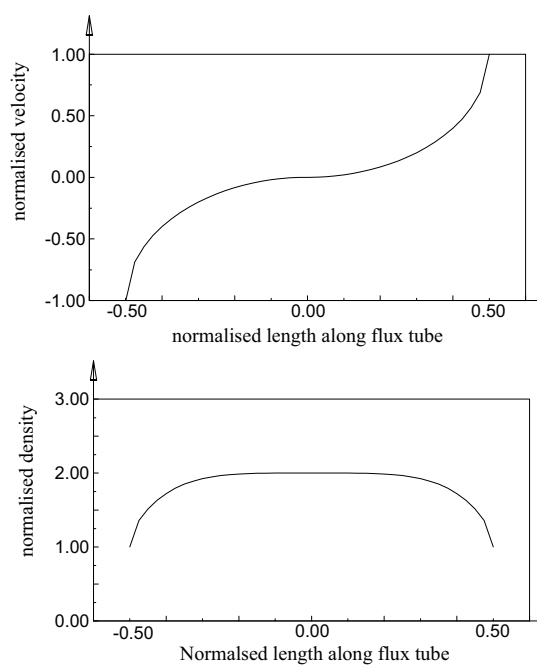

Fig.4: Normalised velocity distribution and density along $a$ flux tube for a constant source function $S(x)$

Fig. 4 shows the normalised velocity (Mach number) and density along the flux tube. As expected, the velocity is zero at the stagnation point and reaches sound velocities at the tube ends. The density at the stagnation point it is twice as high as at the ends. Both density and velocity are rather flat over $80 \%$ of the tube length and fall off rapidly only at the last $10 \%$ on each side.

\section{III.1 PUMP LIMITER}

For power and particle fluxes in the SOL, two flux related exponential decay lengths are important, the particle flux decay length and the power decay length (e.g. $\Gamma=\Gamma_{L C F S} \exp \left(-r / \lambda_{\Gamma}\right)$. The particle flux e.g. towards the limiter is given by $\Gamma=n c_{s}$ leading to

$\lambda_{\Gamma}=\frac{2 \lambda_{n} \lambda_{T}}{2 \lambda_{T}+\lambda_{n}}$

and the power flux by $q=k T n c_{s}$ resulting in

$\lambda_{q}=\frac{2 \lambda_{T} \lambda_{n}}{2 \lambda_{T}+3 \lambda_{n}}$ 
If we take measured data for the ratio as $\lambda_{T} / \lambda_{n}=2$, we obtain $\lambda_{\Gamma}=(4 / 5) \cdot \lambda_{n}$ and $\lambda_{q}=(4 / 7) \cdot \lambda_{n}$. These relations show that the power to a limiter falls off faster than the other decay lengths. This effect is very favourable for constructing a pump limiter. TEXTOR-94 is using such a toroidal pump limiter. A view into TEXTOR and a principal sketch of the cross section of the pump limiter including the pumping section is shown in Figs 5 and 6:

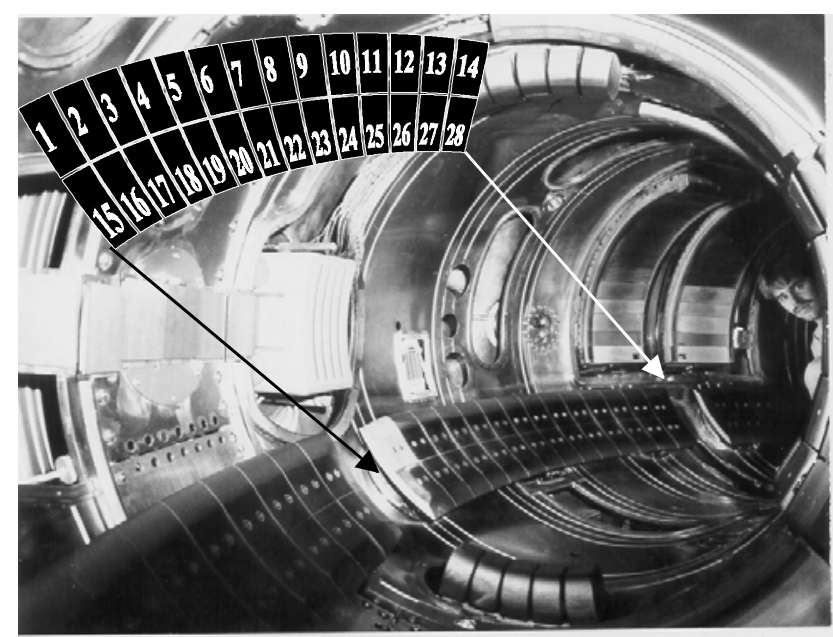

Fig. 5: View into TEXTOR-94 with the toroidal pump limiter ALT-II (surface side)

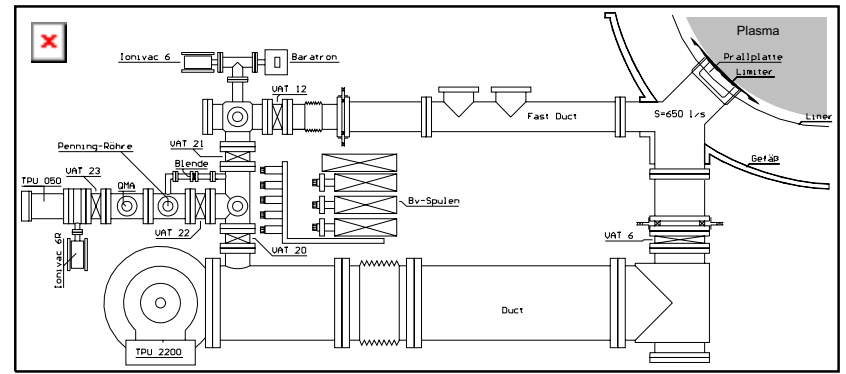

Fig. 6: Sketch of the ALT-II cross section

The major fraction of the power is intercepted by a relatively thin blade. The wider stream of particles flows partly behind the blade and is intercepted by a scoop. At the end of the scoop, the incoming ions are neutralised and scattered into the pumping duct. These particles are either pumped by turbo pumps or escape back through the front opening of the scoop to the plasma. If we put numbers of measured quantities in our estimations we find: $\lambda_{n}=14$ $\mathrm{mm} ; \lambda_{T}=28 \mathrm{~mm}, \lambda_{\Gamma}=11.2 \mathrm{~mm}$ and $\lambda_{q}=8 \mathrm{~mm}$. The pump limiter blade is $20 \mathrm{~mm}$ thick and is covered with graphite tiles (robust low $\mathrm{Z}$ material with high evaporation temperature). Therefore $\left[1-\exp \left(-\Delta r / \lambda_{q}\right)\right]=92 \%$ of the incoming power falls on the limiter blades while $17 \%$ of the particles flow into the scoop. If $50 \%$ of these particles are pumped and the other $50 \%$ escape through the scoop, the removal efficiency of the toroidally symmetric pump limiter ALT-II of TEXTOR amounts to $8.5 \%$. These design values of ALT-II are in agreement with the measurements. We have also verified experimentally the expected helium removal efficiency [10]. The removal efficiencies we obtained for TEXTOR would just be sufficient for the requirements of helium removal for a burning reactor.

\section{III.2. DIVERTORS}

Divertors are created by the magnetic field of external electric currents interacting with the equilibrium magnetic field of the tokamak. Different divertor types are shown in Fig. 7; subfigure a) is the a schematic view of the axisymmetric poloidal divertor, b) is the helical divertor, c) is the toroidal field divertor and d) finally is the bundle divertor.
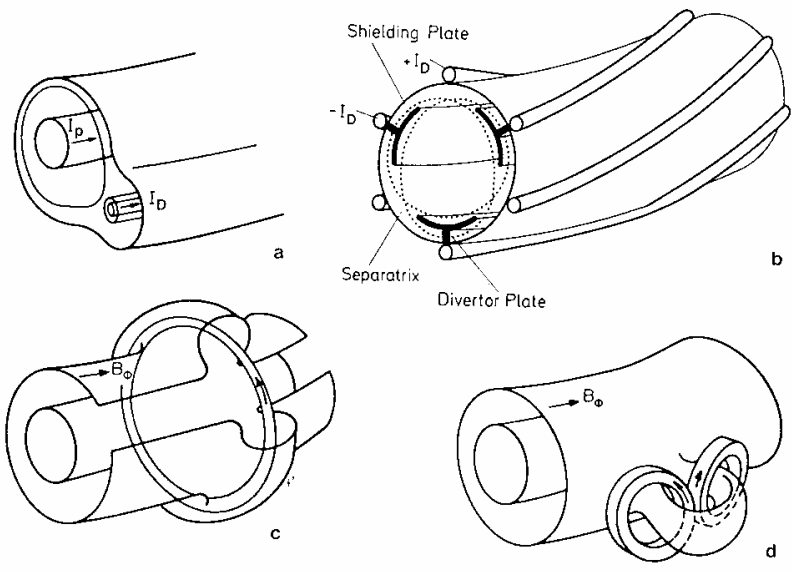

Fig. 7: Different divertor configurations. a) gives the a schematic view of the axi-symmetric poloidal divertor, $b$ ) is the helical divertor, $c$ ) is the toroidal field divertor and d) finally is the bundle divertor

The by most commonly used divertor in tokamaks is the axi-symmetric poloidal divertor -the poloidal magnetic field component is diverted - in which the external current runs in toroidal direction. In this case the poloidal magnetic field component is deflected such that a separatrix is created and the field outside the separatrix is guided into a separate divertor chamber. Power and particles are guided along the magnetic field and inside the divertor chamber the plasma hits a divertor target plate; there the particles are neutralised and deposit their power. In contrast to the pump limiter, power fluxes and particle fluxes must not be treated separately; the divertor chamber is connected with a powerful pump which removes the incoming particles (e.g. the helium ash). 


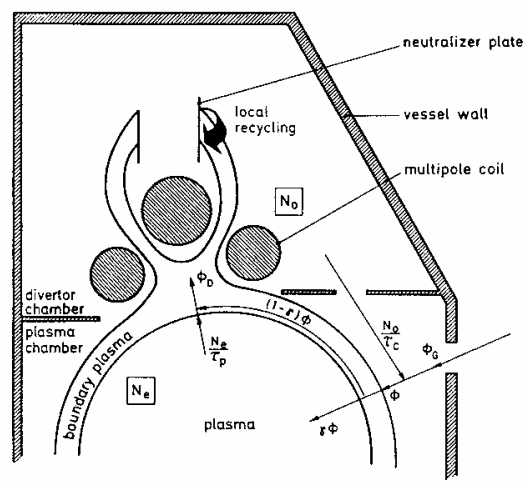

Fig. 8: The divertor configuration of ASDEX is a closed divertor of the first generation; it has paved the way to a new high confinement regime, the H-mode.

A particularly good plasma performance has been reached, if the divertor is closed against particles which have been neutralised at the divertor target plate. An example of this divertor type is the earlier ASDEX divertor which is shown in Fig. 8. In this configuration, the diverting coils were very close to the plasma column. The good closure obtained by the different baffle plates enhances the pumping efficiency just as it has been done by the scoop configuration of the pump limiter. The baffles were installed in order to reduce the back-flow of particles from the divertor chamber, to enhance the pressure there -in order to ease particle pumping - and to remove the impurities from the discharge.

The axi-symmetric divertors have fulfilled the expectations on particle compression and removal; another property which was not foreseen, turned out to be even more important for the success of the poloidal divertor: The divertor, in particular the closed divertor, enables a high confinement regime with strong additional heating. This mode of operation is called the H-mode (high confinement mode in contrast to the L-mode, the low or standard confinement mode). One of the early examples of the enhancement of the energy confinement time with divertor operation is shown in Fig. 9 b (open circles for H-mode).

For large devices like JET or ITER, the strict closing of the divertor and the use of close divertor coils will be impossible (in particular in case of ITER the neutrons would make the use of superconductive coils impossible). Fortunately, the mechanical closing of the divertor chamber is not as necessary as for smaller devices because the plasma can transfer momentum of the flow to the divertor plasma. The scale length of the momentum transfer is the collision length $\lambda_{\text {coll }}$ for $90^{\circ}$ particle scattering which is given by:

$\lambda_{\text {coll }} \approx 5 \cdot 10^{16} \frac{T^{2}(\mathrm{eV})}{n}$
Fig. 10 shows the mean free path $\lambda_{\text {coll }}$ as a function of the density $n$ and temperature $T$.

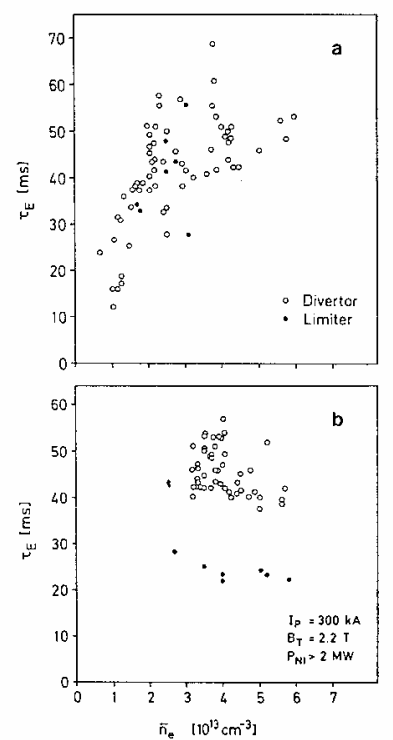

Fig. 9: Energy confinement for ohmic (a) and strongly heated discharges (b). The limiter operation is represented by the closed symbol and the divertor operation by the open circle.

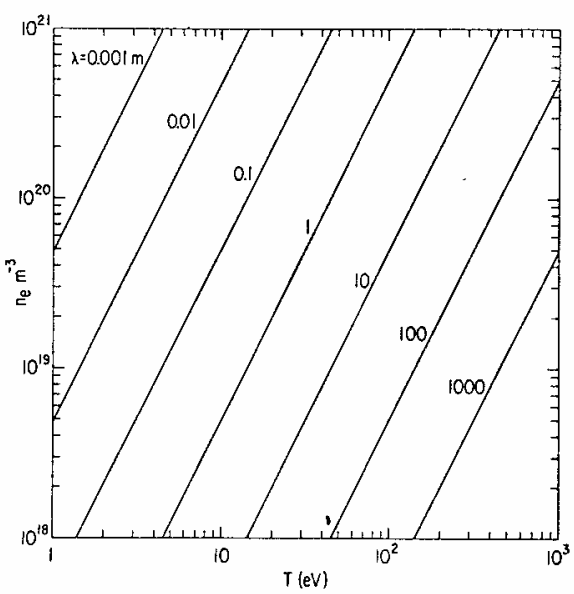

Fig. 10: Mean free path for $90^{\circ}$ scattering as a function of $n$ and $T$.

The curves can be read in different ways. One interpretation is that the fluid approximation of the plasma breaks down, if its scale length is smaller than the collision length; in this case the kinetic treatment of the plasma is mandatory. The other application of the curves is relevant for the momentum exchange. For an edge plasma of $n \approx 10^{18} \mathrm{~m}^{-3}$ and a temperature of $\mathrm{T} \approx 10 \mathrm{eV}$ the collision length 
amounts to about $5 \mathrm{~m}$. To prevent the escape of particles from the divertor zone, i.e. to provide a good particle compression, the good closing of the divertor chamber is required (low recycling case). If, however, the electron temperature is $2 \mathrm{eV}$ and the density amounts to $n \approx 5 \cdot 10^{18} \mathrm{~m}^{-3}$, then the collision length is only a few centimetres long and the momentum from the incoming plasma is easily transferred to the divertor plasma. In this way, the high density plasma can be maintained and frequent collisions between plasma and wall cool the plasma to a sufficiently low level such that sputtering processes are avoided. The neutral particles returning from the wall are easily re-ionised by the dense plasma such they can form a high recycling plasma layer. Many aspects of the high recycling divertor are complex and can be solved by large numerical codes only.

Helical divertors require helical external coils. The helical symmetry appears quite naturally in Stellarators. Therefore helical divertors are foreseen for W7-X; in tokamaks with external perturbation coils for ergodization they have also been considered. The helical external electrical currents impose so called magnetic islands near the plasma boundary. Within certain limits of the perturbation field, these islands form closed flux surfaces similar to the confinement flux surfaces.

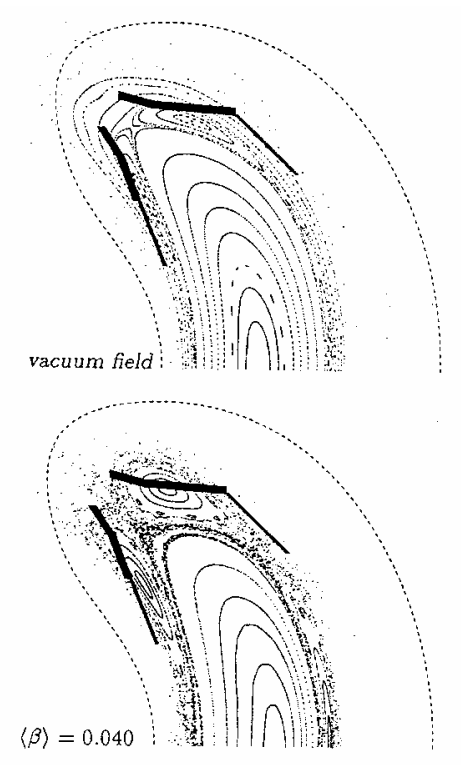

Fig. 11: The proposed W7-X island divertor.

It has been proposed to locate a pump limiter like structure inside the islands and thus to intersect the magnetic flux surfaces. Particles diffusion out of the core are guided along the outer flux tubes as shown in Fig. $7 \mathrm{~b}$ and are neutralised in a scoop like structure. The blade of this helical divertor/pump limiter, which is in the centre of the island, is protected. In the $\mathrm{W} 7-\mathrm{X}$ proposal, the magnetic surfaces of the island will be intersected as shown in Fig. 11: Cooled divertor target plates intercept the power flux from the divertor arriving along the magnetic field lines. The neutralised particles can leave the experimental device through openings where they are pumped. Even though the island divertor looks very open, the island magnetic field lines provide some "magnetic" closing such that also a high recycling operation with the favourable low electron temperatures appears possible.

Bundle divertors and toroidal divertors require very high magnetic fields because the main field has to be deflected. In addition they impose severe disturbances of the confinement field. Therefore they have only very rarely been applied and without positive results.

\section{OBLIQUE TARGET SURFACES}

The sheath theory has been introduced in previous contributions. The important result of these considerations is a) the Bohn criterium for the particle flux $\left(\mathrm{v}_{\text {target }}=\mathrm{c}_{\mathrm{s}}\right)$, and $\mathrm{a}$ criterium for the electric field in the sheath which allows the calculation of the sheath transmission factor for the power. The consideration of the electrostatic sheath hold strictly only if the target is perpendicular to the magnetic flux tubes. In most cases, however, the limiter or divertor target surface is tilted very obliquely in order to distribute the incoming power flux to a large area. Typical angles of incidence are ten degrees reaching down to less than 1 degree. For these small angles of incidence, a new feature, the "magnetic pre-sheath" appears as sketched in Fig. 12. It is composed of two parts, the proper sheath with a length scale of the Debye length for the electrons and the magnetic pre-sheath with a length scale of the ion Larmor radius, i.e. of the order of a $\mathrm{mm}$. The voltage drop derived for the classical sheath now splits up in a contribution $V_{m s}$ of the magnetic pre-sheath and the sheath $V_{s}$. The reason for the creation of the magnetic pre-sheath is that the gyroorbit of the ions is perturbed by the target while the electrons follow the magnetic field line much closer to the target due to their small gyro-radius. The consequence is that the Bohm criterium $\left(\mathrm{v}_{/ / B}=c_{s}\right)$ has now to be applied at the magnetic pre-sheath edge. Within the magnetic pre-sheath, the flow is turned toward the target such that at the electrostatic sheath edge $\mathrm{v}_{\perp T}=c_{s}$. Here $\mathrm{v}_{/ / B}$ and $\mathrm{v}_{\perp T}$ denote the components of the flow velocity parallel to $B$ and normal to the target, respectively.

Equating the ion flux at the magnetic pre-sheath edge (mse) with the electron flux at the electrostatic sheath edge (se), again assuming an ambipolar flow, and the Boltzmann relation for the electrons

$n_{m s e} c_{s} \cos (\Psi)=n_{s e} c_{s}=n_{m s e} \exp \left[e V_{m s} / k T_{e}\right] c_{s}$ 
yields

$e V_{m s} / k T_{e}=\ln (\cos \Psi)$.

For example for $\Psi=80^{\circ}$, one has $e V_{m s} / k T_{e} \approx-1.75$ and hence $e V_{s} / k T_{e} \approx-1.25$ in the electrostatic sheath. The extension of the sheath theory to very small angles of inclination is presently a topic of research.

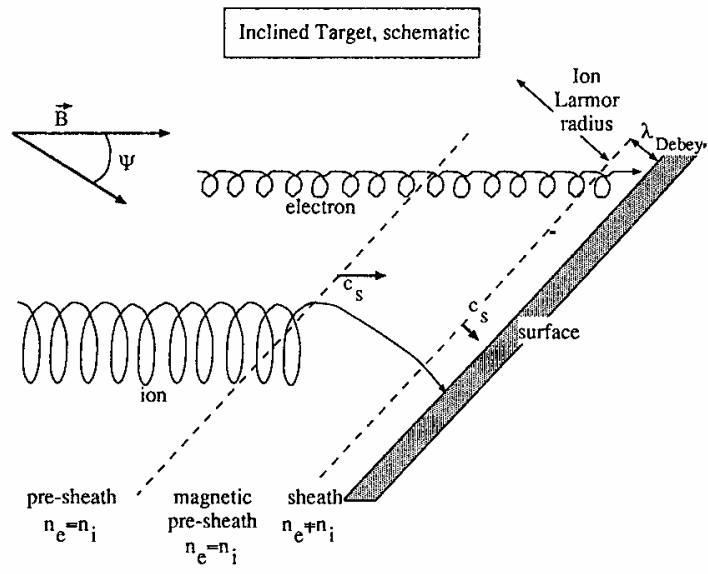

Fig. 12: Inclined target with magnetic pre-sheath and electrostatic sheath.

\section{OUTLOOK AND CONCLUSIONS}

The SOL is treated here in a rather simplified approximation. As already stated before, even this simple model describes many features of the SOL of a limiter device or a divertor at low densities. At high densities, ionisation, charge exchange and recombination effects come more and more into play. In the high recycling regime, the source function is highest near the target plates such that the SOL density along a flux tube in the main tokamak chamber is rather flat and the flow velocity is small. Rather complex is also the flow of impurities in the SOL which are subjected to viscous or thermal forces acting along the flux tubes. Depending on the exact temperature gradient and flow velocity, impurities can either be swept towards the divertor target plate or towards the main plasma chamber. Flow reversals, in particular in divertors but at high densities also in pump limiter scoops, are another important issue. Flow reversals in the SOL result from the radially nonuniform momentum transfer (flux tubes closer to the tangent line carry more particles at higher temperature and have therefore a higher pressure than more remote ones) and the pressure established by the re-ionised particles in the divertor chamber which may be more uniformly distributed than the incoming flux; the superposition of both pressures may force the flow back to the main chamber at some flux tubes. However, all these complicated processes can be treated only numerically in a satisfying way.

In our present understanding, the simultaneous requirement of particle exhaust and power handling in the SOL remains a critical issue for a future reactor. Means to mitigate the power problem are the radiative mantle by seed impurities, the expansion of the magnetic flux tubes in a divertor, the inclination of the target tiles to very shallow angles or the sweeping of the divertor footprints on the target plates. An additional path will be tried in TEXTOR-94 for distributing the heat to a large area by the Dynamic Ergodic Divertor which will be treated in the next contribution.

\section{REFERENCES}

${ }^{1}$ Reiter D., "Helium removal", this volume

${ }^{2}$ Stangeby, P.C., "The Plasma Boundary of Magnetic Fusion Devices", Plasma Physics Series, IOP Publishing Ltd, Bristol, 2000

${ }^{3}$ Picher, C.S., Stangeby, P.C., "Experimental Divertor Physics", Plasma Physics and Controlled Fusion, 39 (1997) 779

${ }^{4}$ Chodura R., "Plasma Flow in the Sheath and Presheath of a Scrape-off Layer" in 'Physics of Plasma Wall Interaction in Controlled Fusion Devices' Post D.E. and Behrisch R. editors, Plenum Press, New York, p. 99 (1986)

${ }^{5}$ Post D.E., Lackner K, "Plasma Models for Impurity Control Experiments", ditto p. 629

${ }^{6}$ Stangeby, P.C., "The Plasma Sheath", ditto p. 41

${ }^{7}$ Stangeby, P.C. and McCracken G.M., "Plasma Boundary Phenomena in Tokamaks", Nucl. Fusion, Vol. 30, P. 1225 (1990)

${ }^{8}$ Gray, D.S. et al., "Plasma Exhaust and Density Control", Nucl. Fusion, Vol. 38, P. 1585 (1998)

${ }^{9}$ Finken, K.H. et al., "Measurements of the Edge Power Flow to the ALT-II Limiter in TEXTOR", J. Nucl. Mater., Vol. 196-198, P., 220 (1992)

${ }^{10}$ Hillis D.L. et al., "Helium Exhaust and Transport Studies with the ALT-II Pump Limiter in the TEXTOR Tokamak", Phys. Rev. Lett., Vol. 65, P. 2382 (1990) 